\title{
O.S.P.
}

L'orientation scolaire et professionnelle

$46 / 1$ | 2017

Adolescence et orientation (1)

\section{Introduction Adolescence et orientation}

Lyda Lannegrand-Willems et Emmanuelle Vignoli

\section{(2) OpenEdition}

\section{Journals}

Édition électronique

URL : https://journals.openedition.org/osp/5309

DOI : 10.4000/osp.5309

ISSN : 2104-3795

Éditeur

Institut national d'étude du travail et d'orientation professionnelle (INETOP)

Édition imprimée

Date de publication : 15 mars 2017

ISSN : 0249-6739

Référence électronique

Lyda Lannegrand-Willems et Emmanuelle Vignoli, «Introduction Adolescence et orientation », L'orientation scolaire et professionnelle [En ligne], 46/1 | 2017, mis en ligne le 01 mars 2019, consulté le 04 janvier 2023. URL : http://journals.openedition.org/osp/5309; DOI : https://doi.org/10.4000/osp. 5309

Ce document a été généré automatiquement le 4 janvier 2023.

Tous droits réservés 


\title{
Introduction Adolescence et orientation
}

\author{
Lyda Lannegrand-Willems et Emmanuelle Vignoli
}

1 " Période de changements à tous niveaux, l'adolescence est caractérisée par des tâches développementales à accomplir, qui, entre besoin individuel et attentes socioculturelles, marquent l'évolution et les changements nécessaires dans les rapports à soi, à autrui et au monde social. Ces changements s'expriment en une variété d'ajustements. L'adolescence se caractérise ainsi par l'émergence de nouvelles conceptions de soi, de l'environnement, et d'exploration et de découvertes de nouvelles possibilités dans différents domaines de vie. C'est le cas particulièrement, dans le domaine de l'orientation scolaire et professionnelle. L'appel à contributions porte sur la thématique de l'adolescence, de la diversité de ses expressions, changements et ajustements, en lien avec les questions d'orientation. »

2 C'est en ces termes que nous avons lancé en juin 2015 un appel à contributions sur le thème "Adolescence et orientation ». Le dernier numéro thématique de la revue L'Orientation Scolaire et Professionnelle consacré à l'adolescence était déjà ancien, il datait de plus de dix ans, paru en 2004, coordonné par F. Bariaud et B. Dumora, et intitulé : «Les adolescents dans la société d'aujourd'hui ». L'étude et la connaissance des processus psychologiques et développementaux en jeu au cours de la période de l'adolescence apportent, cependant, un éclairage spécifique permettant de mieux comprendre les mécanismes psychologiques des conduites d'orientation scolaire et professionnel. Il nous a, en conséquence, semblé essentiel de redonner priorité à cette thématique en lançant un appel à contributions centré sur la question des rapports entre adolescence et orientation.

3 Ce thème reste pleinement d'actualité au sein de la communauté scientifique, en témoigne le nombre de propositions reçues par la revue, près d'une trentaine, et la diversité des approches avec laquelle ces propositions abordent la question de l'orientation et de ses rapports avec la période adolescente. Le succès de cet appel permettra au bout du compte la publication de deux volumes de la revue sur ce thème. 
4 Le présent numéro, premier de ces deux volumes, par les contributions respectives qui le composent, prolonge le numéro thématique précédent édité en 2004, en soulignant et en étudiant comment la question de l'orientation à l'adolescence est liée à la construction du rapport à soi et à la société et de leur articulation. Dans le premier article, H. Lehalle resitue la question des engagements professionnels dans le champ des considérations idéologiques qui se construisent à l'adolescence en analysant la diversité et la complexité de ces changements d'un point de vue développemental. Dans une approche complémentaire centrée sur la dialectique des processus identitaires de socialisation, F. Fourchard et ses collaborateurs proposent dans le deuxième article une réflexion visant à articuler la question de la citoyenneté, en matière d'engagement civique et de construction identitaire à l'adolescence, à celle de l'orientation. Les deux premières contributions constituent des réflexions globales sur certains enjeux psychosociaux du développement à l'adolescence. Dans le troisième article, C. Loisy et E. Carosin apportent une réflexion théorico-pratique centrée sur le pouvoir d'agir en soulignant ses enjeux et implications dans l'accompagnement du développement psychosocial des adolescents. Les deux articles suivants traitent de situations plus spécifiques d'adolescents à prendre en considération. Dans le quatrième article, P. Sahin et I. Orly-Louis exposent une recherche empirique relative aux intentions professionnelles des adolescents et adolescentes se reconnaissant dans une orientation sexuelle minoritaire. Enfin, F. Nunez-Regueiro propose dans le dernier article de concevoir le décrochage scolaire comme processus de stress, en soulignant l'importance de prendre en compte les croyances de justice et d'auto-efficacité tant dans les considérations théoriques qu'appliquées.

5 La diversité, la complémentarité et la qualité de ces différents articles contribueront, sans nul doute, à alimenter nos réflexions théoriques et pratiques sur les questions de l'orientation, de sa place dans la période et les enjeux psychosociaux de l'adolescence, et de ses formes d'accompagnement à cette période de la vie. 